\title{
ESTUDIOS CROMOSOMICOS EN ESPECIES DE HYPOCHAERIS L. (ASTERACEAE, LACTUCEAE) DE CHILE
}

\section{CROMOSOME STUDIES IN SPECIES OF HYPOCHAERIS L. (ASTERACEAE, LACTUCEAE) FROM CHILE}

\author{
Carlos M. Baeza ${ }^{1}$, Carolina Cabezas ${ }^{1}$, Anass Terrab ${ }^{2}$, Tod Stuessy², Eduardo Ruiz ${ }^{1}$, Maria \\ Negritto $^{1} \&$ Estrella Urtubey ${ }^{3}$
${ }^{1}$ Facultad de Ciencias Naturales y Oceanográficas, Departamento de Botánica, Casilla 160-C, Universidad de Concepción, Concepción, Chile. cbaeza@udec.cl
${ }^{2}$ Institute of Botany, University of Vienna, Rennweg 14, A-1030 Wien, Austria.
${ }^{3}$ División Plantas Vasculares, Museo de La Plata, Paseo del Bosque, La Plata, CA. P. 1900, Argentina.

\begin{abstract}
The genus Hypochaeris (Asteraceae, Lactuceae) contains twelve species in Europe, three in Asia, and approximately 50 in South America. In this paper, we worked with mitotic chromosomes of three different species of Hypochaeris from Chile: $H$. acaulis, $H$. incana and $H$. scorzonerae. The species are very different in morphology and geographic distribution. The aim of this work was to compare the chromosome morphology of these species. We found that the chromosomes are different in size and morphology in $H$. acaulis (smaller than $6 \mu \mathrm{m}$ and only chromosome 3 having a secondary constriction on the short arm). In $H$. incana and $H$. scorzonerae the chromosomes are very similar in size, and chromosomes 2 (on the long arm) and 3 (on the short arm) contain secondary constrictions. A tetraploid is reported for $H$. scorzonerae.
\end{abstract}

Hypochaeris L. (Asteraceae, Lactuceae) incluye hierbas anuales o perennes con látex, con hojas arrosetadas, enteras, dentadas o partidas, con corolas amarillas, anaranjadas o blancas (Cabrera 1971, 1974). En la actualidad, se reconocen algo más de 60 especies de Hypochaeris en el mundo, de las cuales 50 se encuentran confinadas a América del Sur, 12 especies a Europa y 3 especies a Asia (SiljakYakovlev et al. 1994; Bortiri, 1999; Baeza et al.2000; Samuel et al. 2003; Weiss et al. 2003a, b; Stuessy et al. 2004). Es un género que muestra una variabilidad morfológica muy grande, especialmente en los caracteres vegetativos, encontrándose desde especies muy pequeñas (H. acaulis) a otras muy grandes (H. scorzonerae) (Weiss et al. 2003a) lo cual ha producido muchas confusiones taxonómicas (Cabrera 1963, 1974, 1976; Wulff 1992; Bortiri 1997; Baeza et al. 2000). Su distribución en Chile es muy amplia extendiéndose desde el nivel del mar hasta sobre los 3000 m (Lack 1979; Weiss et al. 2003a, b), lo que refleja un patrón de radiación adaptativa (Weiss et al. 2003b).
Hypochaeris acaulis (Remy) Britton es una planta pequeña, no mayor de $5 \mathrm{~cm}$ de altura, acaule, con una roseta de hojas muy característica, formando cojines densos. Es parte de la flora andina de América del Sur, y se distribuye en Chile y en Argentina. En nuestro país, se encuentra en Los Andes de la VII y VIII Regiones (Tremetsberger et al. 2003). Hypochaeris incana (Hook. et Arn). Macloskie, es una planta perenne, con flores hermafroditas, polinizadas generalmente por abejas. Esta planta tolera ambientes marítimos y está distribuida en la Patagonia de Chile y Argentina (Moore 1983). Hypochaeris scorzonerae (DC.) F.Muell. es una planta perenne, endémica de Chile, robusta de 30 a $40 \mathrm{~cm}$ de altura. Presenta hojas dispuestas en roseta, oblongo-lanceoladas, irregularmente dentadas, de 20 a $30 \mathrm{~cm}$ de largo. Habita localidades arenosas y húmedas de la zona central de Chile y es una especie frecuente en las Regiones IV y V del país (Hoffmann 1978).

Sobre la base a los antecedentes entregados y considerando que las tres especies de Hypochaeris que fueron estudiadas presentan diferencias 
morfológicas claras y que la distribución geográfica es muy definida para cada taxón, el objetivo de esta investigación fue comparar los cromosomas de estas tres especies para determinar si ellos presentaban diferencias morfológicas y morfométricas como las que se observan en el fenotipo de las plantas.

El material recolectado (semillas) de las especies de Hypochaeris analizadas provienen de distintas localidades de Chile y están depositados en el Herbario de la Universidad de Concepción (CONC).

H. acaulis: VIII Región. Prov. de Biobio. Ralco. Reserva Forestal Alto Bio-Bio. Laguna La Mula, Mallín Alado. Vega altoandina. 30-01-2006. 1706 m (37'53'S$\left.71^{\circ} 23^{\prime} \mathrm{W}\right)$. A. Terrab \& C. Baeza 20.

H. incana: XII Región. Prov. de Magallanes, Comuna de San Gregorio, Parque Nacional Pali Aike. 05-II-2006. 134 m (52 04'S-6946’W). A. Terrab, C. Baeza \& E. Domínguez 37.

H. scorzonerae: V Región. Prov. de Valparaíso. Cuesta Balmaceda, entre camino La Pólvora y Quebrada Honda, segunda curva desde la parte alta. 28-10-2005. 252 m (71³7'S-3306'W). C. Baeza \& P. Novoa 4241.

Para el estudio de los cromosomas se utilizó la metodología propuesta por Weiss-Schneeweiss et al. (2007). Los cromosomas se clasificaron de acuerdo a Levan et al. (1964, modificado) y se midieron con el programa "MicroMeasure 3.3" (Reeves 2001). Para la confección de los idiogramas se utilizó el programa Corel Draw 8.0.

Todas las poblaciones analizadas presentan la misma dotación cromosómica, es decir $2 \mathrm{n}=2 \mathrm{x}=8$, todas presentan el mismo set cromosomal, $4 \mathrm{~m}+4 \mathrm{st}$, esto es, dos pares de cromosomas metacéntricos y dos pares subtelocéntricos. Otra característica citológica es la presencia de constricciones secundarias en el brazo largo del cromosoma 2 y en el brazo corto del cromosoma 3 en las especies Hypochaeris incana e H. scorzonerae (Figs. 1c, 1d y 2b, 2c), en cambio, Hypochaeris acaulis sólo presentó la constricción secundaria en el brazo corto del cromosoma 3 (Figs. 1a y 2a). En la población estudiada de $H$. scorzonerae se encontraron también tetraploides en baja frecuencia (Fig. 1b), lo que ya había sido reportado por Weiss et al. (2003a). Este tipo de tetraploide infraespecífico $(2 \mathrm{n}=4 \mathrm{x}=16)$ ya había sido observado en $H$. apargioides Hook. \& Arn., $H$. taraxacoides (Meyen \& Walp.) Ball e H. tenuifolia (Hook. \& Arn.) Griseb. (Baeza et al. 2006; WeissSchneweiss et al. 2007). La ocurrencia de diploides y tetraploides dentro de estas especies y particularmente dentro de las poblaciones fortalece la hipótesis de la autopoliploidia y sugiere la formación recurrente de poliploides, quizás por medio de gametos no reducidos (Ramsey \& Schemske 1998; Weiss et al. 2002). Hypochaeris acaulis es la única especie que se diferencia claramente de las otras dos por la carencia de una constricción secundaria en el brazo largo del cromosoma 2 y por poseer los cromosomas muy pequeños, no mayores de $6 \mu \mathrm{m}$. Además, es la especie que morfológicamente presenta también el menor tamaño. En cambio, H. incana e $H$. scorzonerae, aunque morfológicamente son muy distintas y su distribución geográfica es característica para cada especie no presentan diferencias en sus cromosomas. Sus cromosomas, con constricciones secundarias en el brazo largo del cromosoma 2 y en el brazo corto del cromosoma 3, también lo presentan otras especies de Hypochaeris sudamericanas, tales como: $H$. alba Cabrera, H. apargioides Hook. \& Arn.,H. brasiliensis (Less.) Benth. \& Hook.f. ex Griseb., H. clarionoides (Remy) Reiche, H. elata (Wedd.) Benth. \& Hook. f. ex Griseb, H. hookeriPhil., H. meyeniana (Walp.) Benth. \& Hook. f. ex Griseb., H. parodii Cabrera, H. sessiliflora Kunth, H. spathulata (Remy) Reiche, H. stenocephala (A. Gray ex Wedd.) Kuntze, H. taraxacoides (Meyen \& Walp.) Ball, H. thrincioides (Remy) Reiche e $H$. variegata (Lam.) Baker (Weiss-Schneeweiss et al. 2007). Es probable que la especiación ocurrida en este grupo haya sido muy rápida debido a una radiación adaptativa hacia muchos hábitats desde el nivel del mar hasta sobre los $2000 \mathrm{~m}$, sin que esta radiación haya sido acompañada por cambios cromosomales conspicuos, situación ya observada en las especies de Dendroseris D. Don y Robinsonia DC. (Asteraceae) del archipiélago de Juan Fernández (Sanders et al. 1987; Crawford et al. 1992).

\section{AGRADECIMIENTOS}

Los autores agradecen el apoyo prestado por el proyecto BBVA: "Erosión genética durante la radiación evolutiva y su impacto sobre los sistemas reproductores en plantas de América Latina: el género Hypochaeris (Asteraceae) como modelo"; a la Fundación Alexander von Humboldt (Georg Foster Stipendium), al proyecto Fundación Andes N ${ }^{\circ} \mathrm{C}-14055$ y al Departamento de Botánica de la Universidad de Concepción por las facilidades otorgadas. 
Estudios cromosómicos en especies de Hypochaeris: BAEZA, C. ET AL.

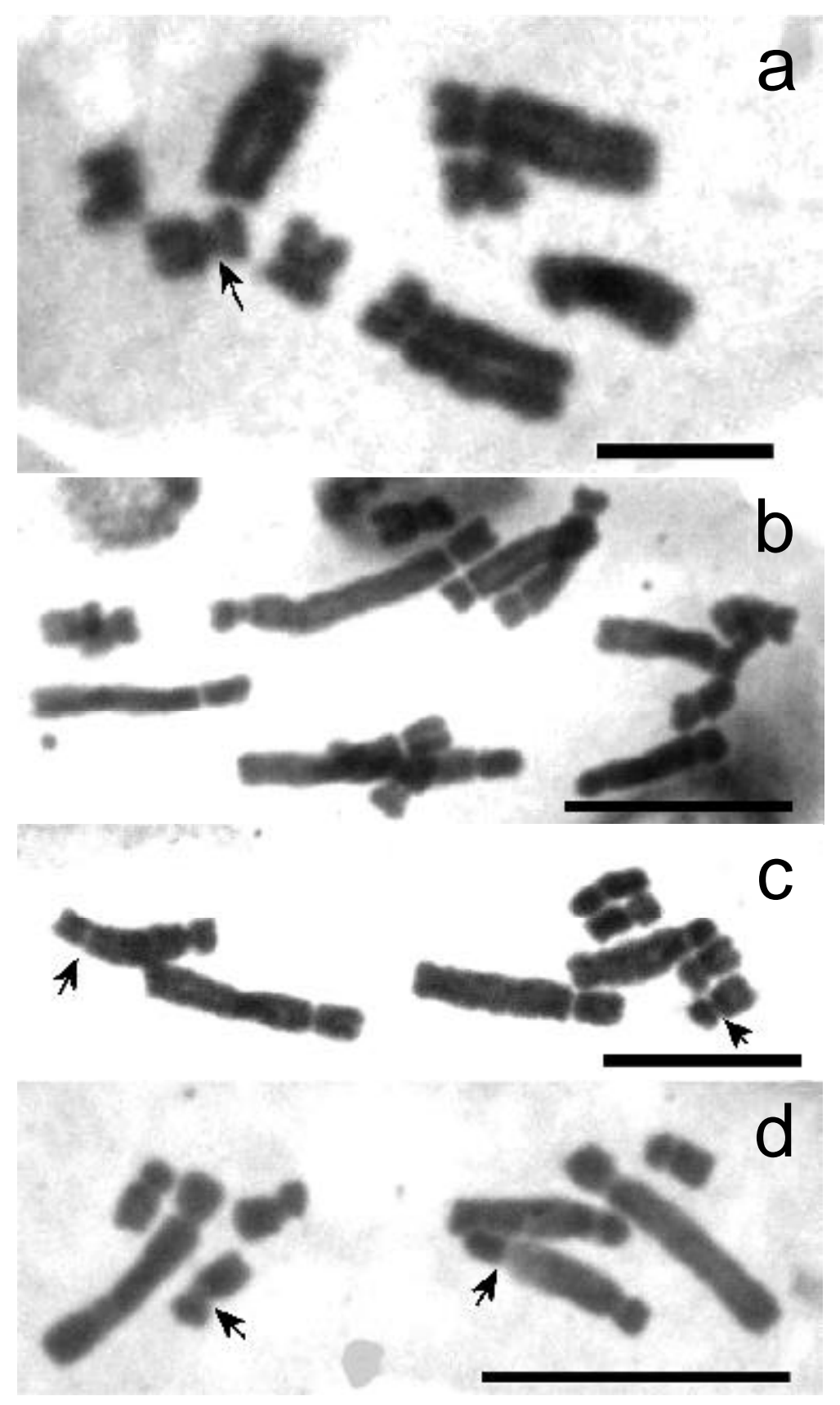

Figura 1. Metafases mitóticas de (a) Hypochaeris acaulis $2 \mathrm{n}=8$ (A. Terrab \& C. Baeza 20. La flecha indica la posición de la constricción secundaria en el cromosoma 3), (b) Hypochaeris scorzonerae $2 \mathrm{n}=16$ (C. Baeza \& P. Novoa 4241), (c) Hypochaeris scorzonerae $2 \mathrm{n}=8$ (C. Baeza \& P. Novoa 4241. Las flechas indican la posición de las constricciones secundarias en los brazos 2 y 3), (d) Hypochaeris incana $2 \mathrm{n}=8$ (A. Terrab, C. Baeza \& E. Domínguez 37. Las flechas indican la posición de las constricciones secundarias en los brazos 2 y 3). Barra (a): $5 \mu \mathrm{m}$, (b-d): $10 \mu \mathrm{m}$.

FiguRE 1. Mitotic metaphases of (a) Hypochaeris acaulis $2 \mathrm{n}=8$ (A. Terrab \& C. Baeza 20. The arrow indicates the position of the secondary constriction on chromosome 3), (b) Hypochaeris scorzonerae $2 \mathrm{n}=16$ (C. Baeza \& P. Novoa 4241), (c) Hypochaeris scorzonerae $2 \mathrm{n}=8$ (C. Baeza \& P. Novoa 4241. The arrows indicate the position of the secondary constriction on chromosomes 2 and 3), (d) Hypochaeris incana $2 n=8$ (A. Terrab, C. Baeza \& E. Domínguez 37. The arrows indicate the position of the secondary constriction on chromosomes 2 and 3). Bar (a) $=5 \mu \mathrm{m},(b-d)=10 \mu \mathrm{m}$. 

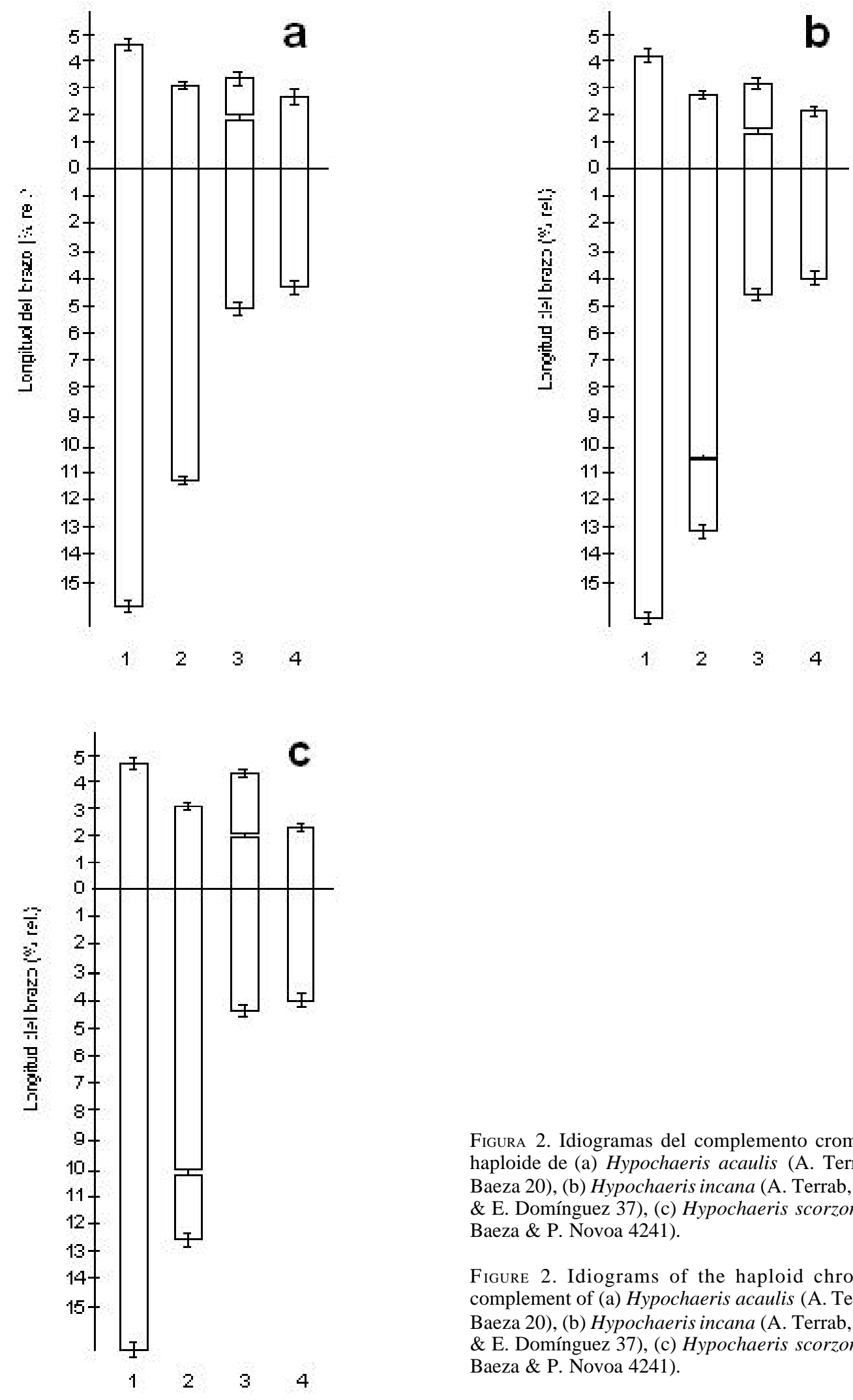

FIgURA 2. Idiogramas del complemento cromosómico haploide de (a) Hypochaeris acaulis (A. Terrab \& C. Baeza 20), (b) Hypochaeris incana (A. Terrab, C. Baeza \& E. Domínguez 37), (c) Hypochaeris scorzonerae (C. Baeza \& P. Novoa 4241).

Figure 2. Idiograms of the haploid chromosome complement of (a) Hypochaeris acaulis (A. Terrab \& C. Baeza 20), (b) Hypochaeris incana (A. Terrab, C. Baeza \& E. Domínguez 37), (c) Hypochaeris scorzonerae (C. Baeza \& P. Novoa 4241). 


\section{BIBLIOGRAFIA}

Baeza, C., J. Grau, M. Vosyka, T. Stuessy \& H. Weiss. 2000. Recuentos cromosómicos en especies de Hypochaeris L. de Chile. Gayana Botánica 57(1): 105-106.

BAEZA, C., S. J ARA \& T. Stuessy. 2006. Cytogenetic studies in Populations of Hypochaeris apargioides Hook. et Arn. (Asteraceae, Lactuceae) from Chile. Gayana Botánica 63(1): 99-105.

Bortiri, E. 1997. Novedades en Hypochaeris (Compositae, Cichorieae) de la Argentina. Hickenia 2(46): 223232.

BortiRi, E. 1999. Asteraceae, parte 14. Tribu XIII. Lactuceae: Hypochoeris. Flora Fanerogámica Argentina 63: 1-25.

CABrera, A.L. 1963. Estudios sobre el género Hypochoeris. Boletín de la Sociedad Argentina de Botánica 10: 166-195.

Cabrera, A.L. 1971. Flora Patagónica, Parte VII. Compositae. Instituto Nacional de Tecnología agropecuaria. Buenos Aires. Argentina.

Cabrera, A.L. 1974. Flora ilustrada de Entre Ríos. Parte VI, Dicotiledoneas Metaclamideas. INTA. Buenos Aires. Argentina. Pp. 512-525.

CABRera, A.L. 1976. Materiales para una revisión del género Hypochoeris. Darwiniana 20: 312-322.

Crawford, D., T.F.Stuessy, M. C osner, D. Haines M. Silva \& C. BAEZA. 1992. Evolution of the genus Dendroseris (Asteraceae: Lactuceae) on the Juan Fernandez Islands: evidence from chloroplast and ribosomal DNA. Systematic Botany 17(4): 676-682.

HofFman, A. 1978. Flora silvestre de Chile. Zona central. Fundación Claudio Gay. 255 pp.

LAck, H. W. 1979. The subtribe Hypochoeridinae (Asteraceae, Lactuceae) in the tropics and the Southern Hemisphere. K. Larsen \& L. B. HolaNielsen (editors), Tropical Botany. Academic Press. London. Pp. 265-276.

Levan, A., Fredga, K. \&A. SAndBerg. 1964. Nomenclature for centromeric position on chromosomes. Hereditas 52: 201-220.

Moore, D. 1983. Flora of Tierra del Fuego. Oswestry, Saint Louis. ix, 396 pp.

Ramsey, J. \& D. Schemske. 1998. Pathways, mechanisms and rates of polyploid formation in flowering plants. Annual Review of Ecology and Systematics 29: 467-501.

ReEves,A. 2001. MicroMeasure: a new computer program for the collection and analysis of cytogenetic data. Genome 44: 239-443.

Samuel, R., T. F. Stuessy, K. Tremetsberger, C. B Aeza, W. Gutterman \& S. Siljak-Yakovlev. 2003.
Phylogenetic relationships among species of Hypochaeris (Asteraceae, Cichorieae) bases on its, plastid trnl intron, trnl/f spacer, and matk sequences. American Journal of Botany 90 (3): 496-507.

Sanders, R., T.F. Stuessy, C. Marticorena \& M. Silva. 1987. Phytogeography and evolution of Dendroseris and Robinsonia, tree-Compositae of the Juan Fernandez Islands. Opera Botanica 92: 195-215.

Siljak - Yakovlev, S.,A. Bartoli, G. Roitman, N. B arghi \& C. Mugnier. 1994. Etude caryologique de tris especes d' Hypochoeris originaires d'Argentine: $H$. chillensis, $H$. microcephala var. albiflora et $H$. megapotanica. Canadian Journal of Botany 72: 1496-1502.

Stuessy, T., K. Tremetsberger, R. Samuel, J. Jankowicz, Y. Guo, A. Muellner \& C. Baeza. 2004. Phylogenetic relationships among South American species of Hypochaeris (Asteraceae) based on aflp data. Plant Evolutionary Genetics and the Biology of Weeds. In B. Schaal, T. Chiang \& C. Chou (Eds.), Endemic Species Research Institute, ChiChi, Taiwan. Pp. 23-39.

Tremetsberger, K., Yan-Ping Guo, H. Weiss, R. Samuel, C. BAeza \& T. Stuessy. 2003.Amplified Fragment Length Polymorphism (AFLP) variation within and among populations of Hypochaeris acaulis (Remy) Britton (Asteraceae) of Andean southern South America. Taxon 52: 237-245.

Weiss, H., C. Dobes, G. Schneeweiss \& J. Greimler 2002. Occurrence of tetraploid and hexaploid cytotypes between and within populations in Dianthus sect. Plumaria (Caryophyllaceae). New Phytologist 156: 85-94.

Weiss, H., T. F. Stuessy, J. Grau, C. Baeza. 2003a. Chromosome reports from South American Hypochaeris. Annals of the Missouri Botanical Garden. 90 (1): 56-63.

Weiss, H., T.F. Stuessy, S. Siljak-Yakovlev, C. Baeza \& J. PARKER. 2003b. Karyotype evolution in the South American species of Hypochaeris (Asteraceae, Lactuceae). Plant Systematics and Evolution 241: 171-184.

Weiss-Schneeweiss, H., T.F.Stuessy, K. Tremetsberger, E. Urtubey, H. Valdebenito, S. Beck \& C. Baeza. 2007. Chromosome numbers and karyotypes of South American species and populations of Hypochaeris (Asteraceae). Botanical Journal of the Linnean Society 153: 49-60.

WuLFF, A. F. 1992. Hibridización natural entre especies Sudamericanas de Hypochoeris (Asteraceae). Darwiniana 31: 167-171. 